Research Paper

\title{
Utility of Plasma Osteopontin Levels in Management of Community-Acquired Pneumonia
}

\author{
Jer-Hwa Chang ${ }^{1,2}$, Wen-Yueh Hung 3 , Kuan-Jen Bai ${ }^{1,2}$, Shun-Fa Yang ${ }^{4,5}{ }^{\bowtie}$, and Ming-Hsien Chien ${ }^{6,7 凶}$ \\ 1. Division of Pulmonary Medicine, Department of Internal Medicine, Wan Fang Hospital, Taipei Medical University, Taipei, Taiwan; \\ 2. School of Respiratory Therapy, College of Medicine, Taipei Medical University, Taipei, Taiwan; \\ 3. Division of Pulmonary Medicine, Department of Internal Medicine, Taipei Medical University Hospital, Taipei, Taiwan; \\ 4. Institute of Medicine, Chung Shan Medical University, Taichung, Taiwan; \\ 5. Department of Medical Research, Chung Shan Medical University Hospital, Taichung, Taiwan; \\ 6. Graduate Institute of Clinical Medicine, Taipei Medical University, Taipei, Taiwan; \\ 7. Department of Medical Education and Research, Wan Fang Hospital, Taipei Medical University, Taipei, Taiwan.
}

$\square$ Corresponding authors: Ming-Hsien Chien, PhD, and Shun-Fa Yang, PhD. Graduate Institute of Clinical Medicine, Taipei Medical University, 250 Wu-Hsing Street, Taipei 110, Taiwan. Phone: +886-2-27361661 ext. 3237; Fax: +886-2-27390500; E-mail: mhchien1976@gmail.com; ysf@csmu.edu.tw

() Ivyspring International Publisher. Reproduction is permitted for personal, noncommercial use, provided that the article is in whole, unmodified, and properly cited. See http://ivyspring.com/terms for terms and conditions.

Received: 2016.05.16; Accepted: 2016.07.27; Published: 2016.08.10

\begin{abstract}
Osteopontin (OPN) is an essential cytokine involved in immune cell recruitment and an important regulator of inflammation. The purpose of this study was to examine differences in OPN plasma levels between before and after antibiotic treatment in hospitalized adult patients with community-acquired pneumonia (CAP). OPN levels were measured in 93 patients with CAP and 54 healthy controls using a commercial enzyme-linked immunosorbent assay (ELISA). The CURB-65, Pneumonia Severity Index (PSI), and Acute Physiology and Chronic Health Evaluation II (APACHE II) scores were used to determine the CAP severity in patients upon initial hospitalization. A decline in the number of white blood cells (WBCs) and neutrophils, and decreases in the levels of OPN and C-reactive protein (CRP) were observed after antibiotic treatment. Only the plasma level of OPN, but not CRP, was correlated with the severity of CAP based on the PSI $(r=0.514, p<0.001)$, CURB-65 $(r=0.396, p<0.001)$, and APACHE II scores $(r=0.473, p<0.001)$. The OPN level also showed a significant correlation with the length of hospital stay $(r=0.210, p=0.044)$. In conclusion, plasma level of OPN may act as diagnostic adjuvant biomarkers for CAP and further play a role in clinical assessment of the severity of CAP, which could potentially guide the development of treatment strategies.
\end{abstract}

Key words: Biochemical marker; Community-acquired pneumonia; Osteopontin; Pneumonia Severity Index.

\section{Introduction}

Community-acquired pneumonia $(\mathrm{CAP})$ is a globally occurring infectious disease with high morbidity, mortality, and costs. In Taiwan, CAP was the fourth leading cause of death in 2014, and the crude mortality rate was 44.2 people per 100,000 population, which accounted for 10,353 deaths [1]. In addition, in the United States, CAP together with influenza was the eighth leading cause of death in 2014 [2]. Since prompt and optimized management of CAP can reduce morbidity and mortality, early diagnosis and recognition of the disease severity are essential for improving the prognosis of CAP patients $[3,4]$.

Osteopontin (OPN) was initially identified as a secreted protein associated with malignant transformation [5]. It is now recognized as a multifunctional phosphorylated acidic glycoprotein that functions as both an extracellular matrix molecule and a major cytokine involved in immune cell recruitment and type-1 (Th1) cytokine expression at sites of inflammation [6,7]. OPN was found to be related to alveolar airspace enlargement with elevated mediators and neutrophilia [8] and plays important roles in cell growth, differentiation, migration, tissue fibrosis, epithelial repair, and regeneration [9-11].

OPN is expressed by a broad range of tissues and cells, for example, epithelial cells, macrophages, $\mathrm{T}$ cells, and fibroblast $[12,13]$. In addition, it may be a 
major mediator of various lung diseases, such as asthma [14], chronic obstructive pulmonary disease [7, 8], emphysema [9], human idiopathic pulmonary fibrosis [15], pulmonary granuloma formation [5], tuberculosis [16], silicosis [17], sarcoidosis [18], influenza [19, 20], and malignancy [5]. In patients suffering from diverse pulmonary diseases, OPN especially seems to be involved in lung inflammation, including interstitial pneumonia [21], sepsis [22], Klebsiella pneumoniae-induced pneumonia [23], eosinophilic pneumonia [24], and pneumococcal pneumonia [25]. However, to our knowledge, no cohort study has explored the prognostic value of OPN in CAP patients. Therefore, we measured plasma levels of OPN in CAP patients and healthy controls to evaluate the value of OPN in predicting the disease severity of CAP patients and to assist the management of CAP patients.

\section{Materials and methods}

\section{Subjects and diagnoses}

Chung Shan Medical University Hospital (CSMUH) is a tertiary care university hospital in Taichung, Taiwan. This cohort study was conducted from January 2009 to December 2012 by the Department of Medical Research and the Departments of Infectious Diseases and Chest Medicine, CSMUH. The study was approved by the Institutional Review Board of CSMUH (IRB no. CS11237). We enrolled 93 CAP patients and 54 healthy controls and all subjects provided informed consent. For the healthy control group, we randomly chose 54 individuals who visited Department of Family Medicine for health examination in the same hospitals from the same geographic area. In addition, subjects with any inflammation or infectious diseases were excluded from control group. For CAP patients, the inclusion criteria consisted of patients aged $>20$ years, who were definitely diagnosed by either emergency room or outpatient department, and who were admitted for treatment of CAP. Demographic characteristics, comorbidities, symptoms and signs of pneumonia, laboratory results, and previous antibiotic treatment were recorded upon admission. The diagnostic criteria for CAP were based on guidelines of the Infectious Diseases Society of America (IDSA)/American Thoracic Society (ATS) [26]. Guidelines for diagnosing CAP included a typical infiltration change on chest $\mathrm{x}$-ray film within 1 day of symptom occurrence and at least one clinical manifestation, such as a cough, yellow thick sputum, or high fever $\left(>37.8^{\circ} \mathrm{C}\right)$; or at least two minor criteria, including tachypnea, dyspnea, pleural pain, chest pain, confusion or disorientation, lung consolidation, or a WBC count of $>12,000$ cells $/ \mathrm{mL}$. Exclusion criteria included being an outpatient; having been transferred from another hospital or having had a separate hospital admission within the previous 3 weeks for other acute conditions such as pulmonary edema, pulmonary embolism, or malignancy appearing during follow-up; pneumonia caused by tuberculosis or malignancy; being severely immunocompromised, including severe neutropenia (with a WBC count of $<10^{9}$ cells/L); and having an organ or bone marrow transplant or human immunodeficient virus infection. All CAP patients were given empirical antimicrobial agents (e.g., moxifloxacin, levofloxacin, and amoxicillin) intravenously within the first $48 \mathrm{~h}$. Thereafter, oral antibiotics were given based on established guidelines. Blood samples before treatment were collected before patients with CAP received treatment protocols, and post-treatment blood samples were obtained within 3 days after the pneumonia had resolved. The collected blood samples were placed in tubes containing EDTA, immediately centrifuged at $2500 \times \mathrm{g}$, stored at $-80^{\circ} \mathrm{C}$, and used to measure WBCs, neutrophils, the CRP level, and OPN plasma level. Pneumonia severity was evaluated by the PSI [27], APACHE II [28], and CURB-65 [29] tests.

\section{Measurements of WBCs, neutrophils, and CRP levels}

WBCs, neutrophils, and CRP levels were measured by clinical laboratory staff members who were unaware of the source for the samples (i.e., blinded to the study).

\section{Quantitative analysis of plasma OPN level}

The OPN levels in the plasma samples were analyzed by human osteopontin enzyme-linked immunosorbent assay (ELISA) kits (ADI-900-142, Enzo). Briefly, $100 \mu \mathrm{L}$ of prepared standards and samples were added to appropriate wells of ELISA plate and then assayed according to the manufacturer's instructions. The absorbance was measured at $450 \mathrm{~nm}$ in a microtest plate spectrophotometer, and OPN levels were quantified with a calibration curve using human OPN as a standard. Each standard or sample was assayed in duplicate.

\section{Statistical analysis}

SPSS 15.0 statistical software (SPSS, Chicago, IL, USA) was used for all statistical analyses. Continuous variables are expressed as mean \pm standard deviation (SD), and the number (n) is presented with percentages for categorical variables. To compare untreated patients with controls, the Mann-Whitney U-test was used for continuous variables that did not 
follow a parametric distribution, and the Wilcoxon signed-rank test was used to compare categorical variables of untreated and treated patients. A linear regression analysis was applied for correlations between OPN and all clinical and laboratory variables of CAP patients. Statistical significance was defined at $p<0.05$ in a two-tailed test.

\section{Results}

Demographic and clinical characteristics are summarized in Table 1. Totally, 147 subjects were included in the analysis, and the age and percentage of males did not significantly differ between CAP patients and controls. Among the 93 CAP patients, the mean scores of the PSI, CURB-65, and APACHE II were $82.97 \pm 37.17,1.09 \pm 0.93$, and $9.94 \pm 5.31$, respectively. The mean hospital length of stay was $11.71 \pm 17.49$ days. CAP patients had significantly higher CRP levels $(11.26 \pm 7.30$ vs. $0.46 \pm 0.27 \mathrm{mg} / \mathrm{dl}$; Table 1), WBCs $(12,505.4 \pm 5533.9$ vs. $6280.7 \pm 1814.5$ cells $/ \mathrm{mm}^{3}$; Table 1), and neutrophils (9904.1 \pm 5010.1 vs. $3670.9 \pm 1341.7$ cells $/ \mathrm{mm}^{3}$; Table 1) compared to control subjects $(p<0.001)$. Moreover, there were significant decreases in CRP levels (untreated: $11.26 \pm$ $7.30 \mathrm{mg} / \mathrm{dl}$; treated: $4.61 \pm 4.65 \mathrm{mg} / \mathrm{dl}$; Table 1), WBCs (untreated: 12,505.4 \pm 5533.9 cells $/ \mathrm{mm}^{3}$; treated: $8752.0 \pm 3673.2$ cells $/ \mathrm{mm}^{3}$; Table 1 ), and neutrophils (untreated: $9904.1 \pm 5010.1$ cells $/ \mathrm{mm}^{3}$; treated: 6265.3 \pm 3402.2 cells $/ \mathrm{mm}^{3}$; Table 1 ) after antibiotic treatment $(p<0.001)$.

Figure 1 shows plasma OPN levels in control subjects and CAP patients before and after antibiotic treatment. CAP patients presented with significantly higher OPN plasma levels compared to control subjects (controls: $9.16 \pm 5.61 \mathrm{ng} / \mathrm{mL}$; patients: $24.32 \pm$

Table 1. Laboratory data of controls and patients with community-acquired pneumonia (CAP) before and after antibiotic treatment a, b

\begin{tabular}{|c|c|c|c|c|}
\hline \multirow[t]{2}{*}{ Clinical variable } & \multirow{2}{*}{$\begin{array}{l}\text { Controls } \\
(n=54)\end{array}$} & \multicolumn{2}{|c|}{ CAP patients $(n=93)$} & \multirow{2}{*}{$\begin{array}{l}p \text { value } \\
\mathrm{UT} / \mathrm{C}^{\mathrm{c}} \mathrm{UT} / \mathrm{T} \mathrm{d}\end{array}$} \\
\hline & & Before treatment & After treatment & \\
\hline Age (years) & $59.57 \pm 11.05$ & $63.15 \pm 20.98$ & & 0.247 \\
\hline \multicolumn{5}{|l|}{ Gender } \\
\hline Male & $36(66.7 \%)$ & $56(60.2 \%)$ & & 0.436 \\
\hline Female & $18(33.3 \%)$ & $37(39.8 \%)$ & & \\
\hline $\mathrm{CRP}(\mathrm{mg} / \mathrm{dl})$ & $0.46 \pm 0.27$ & $11.26 \pm 7.30$ & $4.61 \pm 4.65$ & $<0.001<0.001$ \\
\hline WBCs (cells/mm³) & $6280.7 \pm 1814.5$ & $12,505.4 \pm 5533.9$ & $8752.0 \pm 3673.2$ & $<0.001<0.001$ \\
\hline Neutrophils (cells $/ \mathrm{mm}^{3}$ ) & $3670.9 \pm 1341.7$ & $9904.1 \pm 5010.1$ & $6265.3 \pm 3402.2$ & $<0.001<0.001$ \\
\hline PSI score & & $82.97 \pm 37.17$ & & \\
\hline CURB-65 score & & $1.09 \pm 0.93$ & & \\
\hline APACHE II score & & $9.94 \pm 5.31$ & & \\
\hline Hospital length of stay (days) & & $11.71 \pm 17.49$ & & \\
\hline \multicolumn{5}{|c|}{$\begin{array}{l}\text { CRP, C-reactive protein; WBCs, white blood cells; C, controls; UT, CAP patients before they received } \\
\text { antibiotic treatment; } \mathrm{T}, \mathrm{CAP} \text { patients after they received antibiotic treatment; PSI, Pneumonia Severity } \\
\text { Index; CURB- } 65, \text { confusion, urea of }>7 \mathrm{mmol} / \mathrm{l} \text {, respiratory rate of }>30 / \mathrm{min} \text {, low systolic }(<90 \mathrm{mmHg}) \text { or } \\
\text { diastolic }(<60 \mathrm{mmHg}) \text { blood pressure, and aged } \geq 65 \text { years; APACHE, Acute Physiology and Chronic } \\
\text { Health Evaluation. } \\
\text { a } p<0.05 \text { was considered significant. } \\
\text { b Data were presented as the mean } \pm \mathrm{SD} \text { and } \mathrm{n}(\%) \text {. } \\
\text { c The statistical difference was analyzed by the Mann-Whitney U-test. }\end{array}$} \\
\hline
\end{tabular}

$14.08 \mathrm{ng} / \mathrm{mL} ; p<0.001$; Figure 1). After CAP patients received antibiotic treatment, OPN levels significantly dropped (untreated: $24.32 \pm 14.08 \mathrm{ng} / \mathrm{mL}$; treated: $16.50 \pm 12.01 \mathrm{ng} / \mathrm{mL} ; p<0.001$; Figure 1).

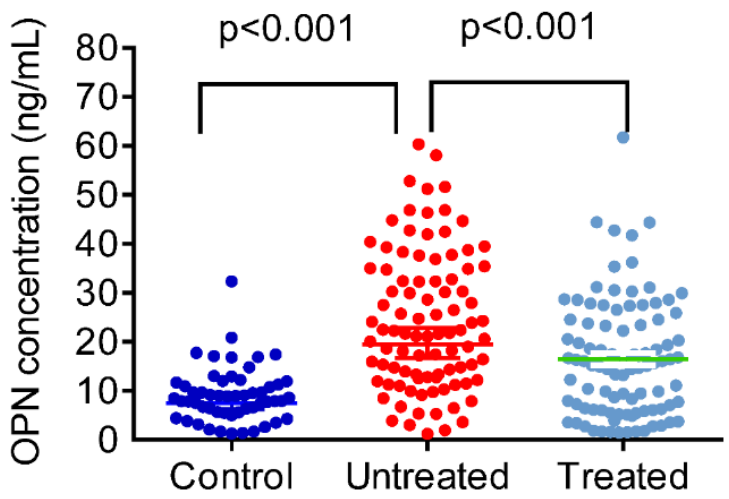

Figure 1 Plasma levels of osteopontin (OPN) in control subjects and patients with ommunity-acquired pneumonia (CAP) before and after treatment. The plasma OPN level was significantly elevated in patients with CAP before they received treatment compared with the controls $(p<0.001)$ and significantly decreased in patients with CAP after treatment $(p<0.001)$. Data were presented as the mean \pm SD. Control vs Untreat: The statistical difference was analyzed by the Mann-Whitney U-test. Untreated vs Treated: The statistical difference was analyzed by the Wilcoxon signed-rank test.

To further examine the correlation between plasma OPN levels and the severity of CAP before treatment, we used the PSI, CURB-65, APACHE II, and the length of hospital stay as pneumonia severity indices. Correlations among them are shown in Table 2. There were significant correlations between OPN and the PSI (Spearman correlation coefficient $\mathrm{r}=$ 0.514, $p<0.001$; Figure 2A), CURB-65 (Spearman correlation coefficient $r=0.396, p<0.001$; Figure 2B), APACHE II (Spearman correlation coefficient $r=$ 0.473, $p<0.001$; Figure 2C). Although the same trend of OPN and CRP were observed in CAP patients before and after antibiotic treatment, there was no significant correlation between OPN levels and CRP levels in CAP patients before (Figure 2D) and after (Figure 2E) antibiotic treatment.

The rule of PSI stratifies CAP patients into five classes of risk for death within 30 days of presentation. The lowest risk class (risk class I) comprises patients who are younger than 50 years of age, have none of the five important coexisting illnesses and have normal mental status and normal or only mildly abnormal vital signs at presentation. Assignment to the remaining risk classes depends on the presence or absence of a set of medical history, physical examination, and laboratory 
findings. Total point scores of 70 or less correspond to class II, 71 to 90 to class III, 91 to 130 to class IV, and more than 130 to class V. Mortality rates in risk classes I, II, and III are low $(0.1 \%$ to $0.4 \%$ in class I and $0.9 \%$ to $2.8 \%$ in class III), with correspondingly medium and high mortality rates in risk classes IV and V, respectively [30]. In our study, we found that OPN levels in CAP patients with risk classes IV and V all significantly differed between classes I and II (Figure $3 \mathrm{~A})$. The OPN levels in CAP patients with medium or high mortality risk were significantly higher than patients with low mortality risk (Figure 3B). Taken together, OPN might be a potential biochemical marker to diagnose the severity of CAP and predict the mortality rate of patients with CAP.
A

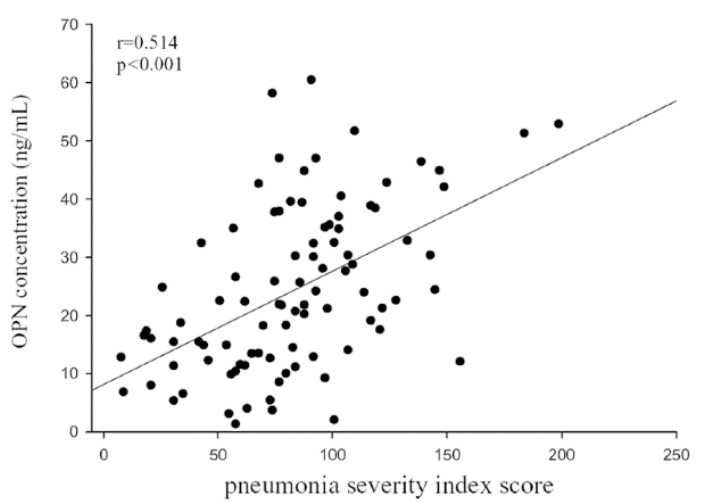

$\mathrm{C}$
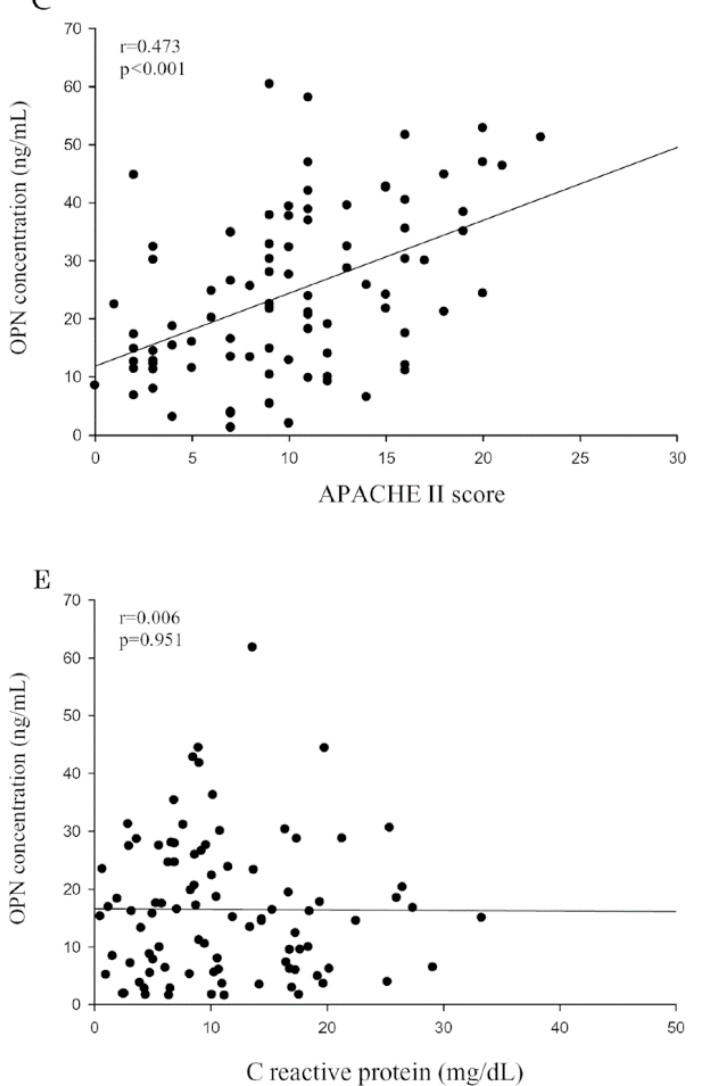

B
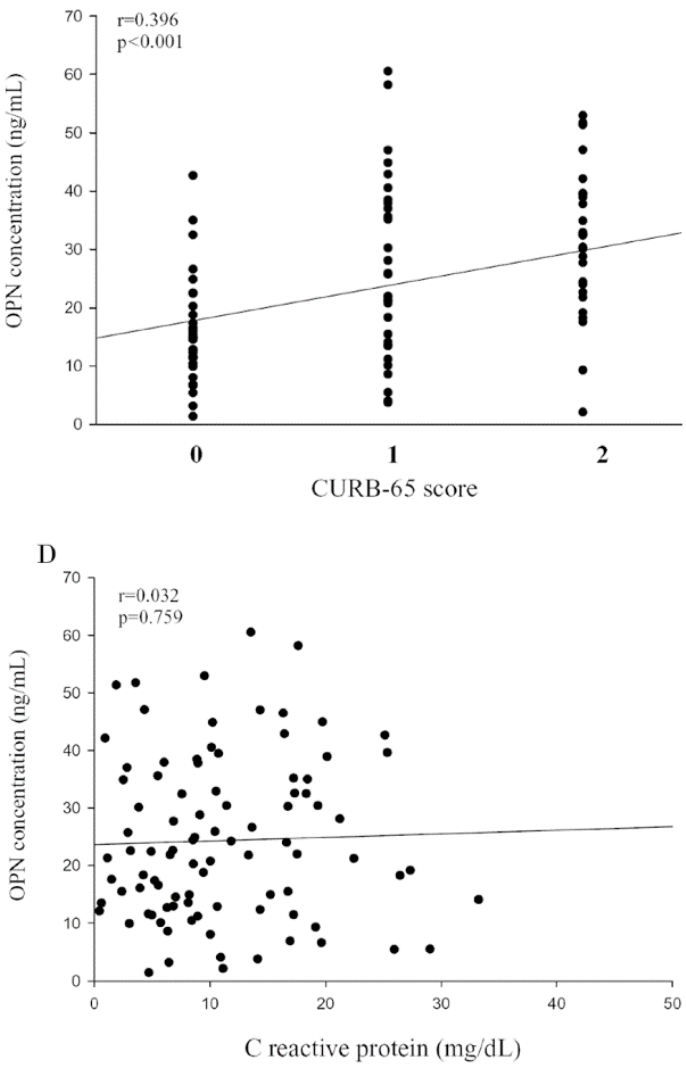

Figure 2 Correlations of plasma osteopontin (OPN) levels with the pneumonia severity index (PSI), confusion, urea level, respiratory rate, blood pressure, and age of $\geq 65$ years (CURB-65), and Acute Physiology and Chronic Health Evaluation II (APACHE II) scores in 93 patients with community-acquired pneumonia (CAP). (A) A significantly positive correlation was observed between plasma OPN levels and PSI scores (Spearman's correlation coefficients: $r=0.514, p<0.001$ ). (B) A significantly positive correlation was observed between plasma OPN levels and CURB-65 scores (Spearman's correlation coefficients: $r=0.396, p<0.001$ ). (C) A significantly positive correlation was observed between plasma OPN levels and APACHE II scores (Spearman's correlation coefficients: $r=0.473, p<0.001$ ). (D and E) The correlation between plasma OPN levels and CRP values were insignificant before antibiotic treatment (Spearman's correlation coefficients: $r=0.032, p=0.759$ ) and after antibiotic treatment (Spearman's correlation coefficients: $r=0.006, p=0.951)$. A linear regression analysis was applied for correlations between OPN and all clinical and laboratory variables of CAP patients. 
A

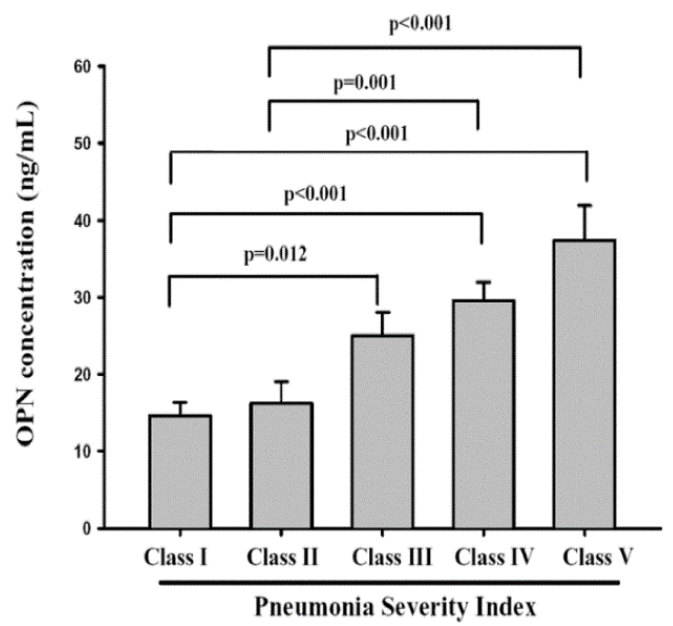

B

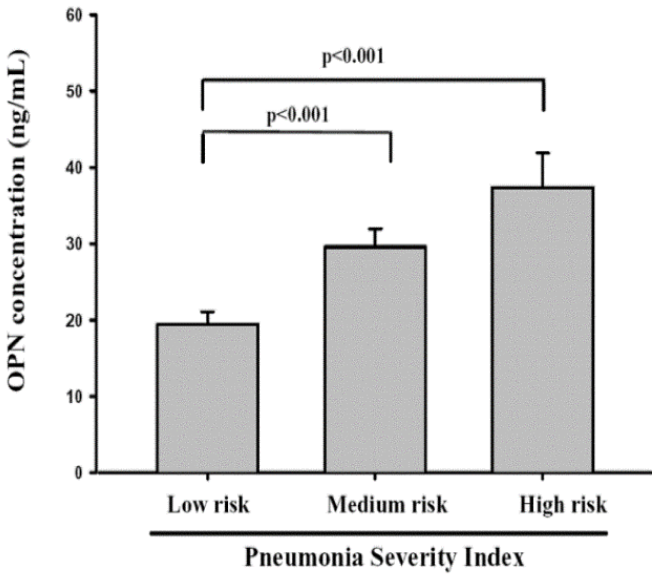

Figure 3 Plasma osteopontin (OPN) levels of community-acquired pneumonia (CAP) patients with each Pneumonia Severity Index (PSI) class (class I, $\mathrm{n}=16$; II, $\mathrm{n}=16$; III, $\mathrm{n}=$ 23; IV, $n=29 ; \mathrm{V}, \mathrm{n}=9$ ) and different mortality risk (low, $\mathrm{n}=55$; medium, $\mathrm{n}=29$; and high, $\mathrm{n}=9$ ). Data were presented as the mean $\pm \mathrm{SD}$. The statistical difference was analyzed by the Mann-Whitney U-test.

In contrast to OPN, neither CRP levels nor WBC counts correlated with the CAP severity in patients, as evidenced from the statistically nonsignificant correlations among PSI, CURB-65, and APACHE II scores and CRP levels $(p=0.755,0.606$, and 0.741, respectively) and $\mathrm{WBC}$ counts $(p=0.921,0.514$, and 0.922 , respectively; Table 2). In addition, we also found that only OPN levels, but not CRP levels and WBC counts correlated significantly with the length of hospital stay (Spearman correlation coefficient $r=$ $0.210, p=0.044$; Table 2).

Table 2. Correlation of white blood cells (WBCs), C-reactive protein (CRP), and osteopontin (OPN) with clinical pathological features of CAP patients.

\begin{tabular}{lllllll}
\hline Variables & \multicolumn{2}{l}{ WBC $(n=93)$} & \multicolumn{2}{l}{ CRP $(n=93)$} & \multicolumn{2}{l}{ OPN $(n=93)$} \\
\cline { 2 - 7 } & $r$ & $p$ & $r$ & $p$ & $r$ & $p$ \\
\hline PSI score & -0.010 & 0.921 & -0.033 & 0.755 & 0.514 & $<0.001$ \\
CURB-65 score & 0.069 & 0.514 & 0.054 & 0.606 & 0.396 & $<0.001$ \\
APACHE II score & -0.010 & 0.922 & 0.035 & 0.741 & $0.473<0.001$ \\
Length of hospital stay & -0.033 & 0.754 & 0.023 & 0.825 & 0.210 & 0.044
\end{tabular}

PSI, Pneumonia Severity Index; CURB-65, confusion, urea of $>7 \mathrm{mmol} / \mathrm{l}$, respiratory rate of $>30 / \mathrm{min}$, low systolic $(<90 \mathrm{mmHg})$ or diastolic $(<60 \mathrm{mmHg})$ blood pressure, and aged $\geq 65$ years; APACHE II, Acute Physiology and Chronic Health Evaluation II.

\section{Discussion}

Our results revealed that: (I) plasma OPN levels in patients with CAP were higher compared to those of healthy controls and significantly declined in the same patients after antibiotic treatment; (II) OPN levels were significantly correlated with disease severity indices; and (III) OPN levels presented a significant relation with the length of hospital stay for CAP.

Significantly higher values of CRP levels, WBCs, and neutrophils in CAP patients before antibiotic treatment, compared to the control group, significantly declined after antibiotic treatment in CAP patients, which reveals their traditional roles in CAP diagnoses. However, some other studies have indicated that elevated WBC counts and CRP levels in patients with CAP have no prognostic relevance [31, 32]. For example, in pneumococcal CAP patients, despite having a high bacterial burden, there were relatively weak CRP responses in the majority of severe cases of pneumonia [33]. CRP levels or WBCs were not significantly related to a change in the clinical classification or mortality of patients with pneumonia [31-34].

In consistent with previous studies, our study showed that no significant correlation was observed between the CRP levels or WBC counts and CAP severity indices (PSI, CURB-65, and APACHE II). In contrast to CRP, results presented here show that plasma OPN levels were higher in patients with CAP compared to those of healthy controls and significantly decreased in the same patients after they received antibiotic treatment. High OPN values were associated with several variables (PSI, CURB-65, and APACHE II) indicative of the disease severity. Several previous reports also suggested that CRP and the disease severity is correlated $[35,36]$. This controversial role of CRP in management of CAP could probably relate to the recruited population size, race, sex, and so on in different studies. Moreover, our study showed that there was no significant correlation 
between OPN levels and CRP levels before and after antibiotic treatment, even if the same trend of CRP and OPN were observed in CAP patients before and after antibiotic treatment. This result suggested that OPN might play a different role with CRP in management of CAP. In addition to disease severity, PSI has been categorized into five risk classes which associate with low (classes I III), medium (class IV), and high (class V) mortality rate of CAP patients [30]. The significant difference between OPN values in CAP patients with medium or high mortality rate and patients with low mortality rate in this study suggests that OPN might be a potential biochemical marker to predict the mortality rate of patients with CAP. Actually, a previous report indicated that OPN serum levels can predict mortality in critically ill patients during the early course of intensive care unit (ICU) treatment [37]. Although both CRP and OPN can be induced by proinflammatory cytokines such as interleukin (IL)-1 $\beta$ and tumor necrosis factor- $\alpha$ [38, 39], CRP is almost exclusively synthesized by hepatocytes under stimulation by inflammatory cytokines [40]. However, OPN especially seems to be involved in lung inflammation, as patients suffering from diverse pulmonary diseases, including interstitial pneumonia [41], tuberculosis, silicosis [17], and acute respiratory distress syndrome [42], have shown enhanced OPN expression in their lungs, whereas patients with idiopathic pulmonary fibrosis have shown increased OPN levels in bronchoalveolar lavage fluid [43]. We suggest that OPN acts as a more direct reflective marker than does CRP with respect to the response to an inflammatory stimulus in lung tissues and also as a more specific marker of the diagnosis and clinical assessment of CAP severity in Taiwanese populations.

Clinical guidelines for managing CAP patients suggest using a severity-based approach for guiding therapeutic options, such as the need for hospital or ICU admission, suitability for ambulatory care, and choice of antimicrobial agents. Although we have found that OPN might be a potential biomarker for predicting the severity and mortality of CAP, but whether OPN can selectively recognize different types of pathogens remains unclear. Identifying the etiology of CAP is clinically difficult because single clinical, radiological, or laboratory parameters have limited value for predicting the infectious organism [44], and no rapid test has been standardized for diagnosing atypical or viral pathogens. Therefore, a type of empirical broad-spectrum antibiotic therapy is typically selected [45]. A previous report indicated that OPN levels were associated with Streptococcus pneumoniae-induced pneumonia in mice [25]. However, the limitation of this study is a lack of more-detailed clinical data, such as information on comorbid diseases and microbial pathogens. Some comorbidities might interfere with plasma OPN levels, and different pathogens might have different impacts on CAP severity. For example, OPN can impair host defense during Streptococcus pneumoniae-induced pneumonia, but promote the host defense during Klebsiella pneumoniae-induced pneumonia $[23,25]$. Future studies are necessary to validate the precise correlation between plasma OPN levels and CAP due to different microbial pathogens. In addition, almost all the CAP patients we recruited in this study were not diagnosed as having severe $\mathrm{CAP}$ requiring intensive care unit admission. We can't get the information about survival data (only four patients died in our recruited patients). We will further investigate the correlation between the OPN levels and survival rate in patients with severe CAP in the future.

In conclusion, plasma OPN levels can be used in Taiwanese populations to predict the severity and mortality of CAP with higher sensitivity compared to CRP and is associated with the effect of antibiotic treatment and the length of hospital stay. In this study, we reported that measuring plasma levels of OPN can be beneficial for clinical decision making in CAP management.

\section{Acknowledgments}

This study was supported by grant numbers 105TMU-TMUH-08 from Taipei Medical University Hospital and 105-swf-07 from Wan Fang Hospital, Taipei Medical University.

\section{Conflict of Interest}

The authors stated that there are no conflicts of interest regarding the publication of this article.

\section{References}

1. MOHW. 2014 statistics of causes of death. Taipei City, Taiwan (R.O.C.): Ministry of Health and Welfare; 2015.

2. Murphy SL, Kochanek KD, Xu J, Arias E. Mortality in the United States, 2014. NCHS Data Brief. 2015; 1-8.

3. Houck PM, Bratzler DW, Nsa W, Ma A, Bartlett JG. Timing of antibiotic administration and outcomes for Medicare patients hospitalized with community-acquired pneumonia. Arch Intern Med 2004; 164: 637-44.

4. Kanwar M, Brar N, Khatib R, Fakih MG. Misdiagnosis of community-acquired pneumonia and inappropriate utilization of antibiotics: side effects of the 4-h antibiotic administration rule. Chest 2007; 131: 1865-9.

5. O'Regan A. The role of osteopontin in lung disease. Cytokine Growth Factor Rev 2003; 14: 479-88.

6. Chabas D, Baranzini SE, Mitchell D, Bernard CC, Rittling SR, Denhardt DT, et al. The influence of the proinflammatory cytokine, osteopontin, on autoimmune demyelinating disease. Science 2001; 294: 1731-5.

7. Papaporfyriou A, Loukides S, Kostikas K, Simoes DC, Papatheodorou G, Konstantellou E, et al. Increased levels of osteopontin in sputum supernatant in patients with COPD. Chest 2014; 146: 951-8.

8. Schneider DJ, Lindsay JC, Zhou Y, Molina JG, Blackburn MR. Adenosine and osteopontin contribute to the development of chronic obstructive pulmonary disease. FASEB J 2010; 24: 70-80.

9. Shan M, Yuan X, Song LZ, Roberts L, Zarinkamar N, Seryshev A, et al. Cigarette smoke induction of osteopontin (SPP1) mediates $\mathrm{T}(\mathrm{H}) 17$ 
inflammation in human and experimental emphysema. Sci Transl Med 2012; 4: $117 \mathrm{ra9}$.

10. Di Stefano A, Caramori G, Gnemmi I, Contoli M, Vicari C, Capelli A, et al. T helper type 17-related cytokine expression is increased in the bronchial mucosa of stable chronic obstructive pulmonary disease patients. Clin Exp Immunol 2009; 157: 316-24.

11. Hillas G, Loukides S, Kostikas K, Simoes D, Petta V, Konstantellou E, et al. Increased levels of osteopontin in sputum supernatant of smoking asthmatics. Cytokine 2013; 61: 251-5.

12. Brown LF, Berse B, Van de Water L, Papadopoulos-Sergiou A, Perruzzi CA, Manseau EJ, et al. Expression and distribution of osteopontin in human tissues: widespread association with luminal epithelial surfaces. Mol Biol Cell 1992; 3: 1169-80.

13. O'Regan AW, Chupp GL, Lowry JA, Goetschkes M, Mulligan N, Berman JS. Osteopontin is associated with T cells in sarcoid granulomas and has $\mathrm{T}$ cell adhesive and cytokine-like properties in vitro. J Immunol 1999; 162: 1024-31.

14. Delimpoura V, Bakakos P, Tseliou E, Bessa V, Hillas G, Simoes DC, et al. Increased levels of osteopontin in sputum supernatant in severe refractory asthma. Thorax 2010; 65: 782-6.

15. Kato A, Okura T, Hamada C, Miyoshi S, Katayama H, Higaki J, et al. Cell stress induces upregulation of osteopontin via the erk pathway in type II alveolar epithelial cells. PLoS One. 2014; 9: e100106.

16. Koguchi Y, Kawakami K, Uezu K, Fukushima K, Kon S, Maeda M, et al. High plasma osteopontin level and its relationship with interleukin-12-mediated type $1 \mathrm{~T}$ helper cell response in tuberculosis. Am J Respir Crit Care Med 2003; 167: 1355-9

17. Nau GJ, Guilfoile P, Chupp GL, Berman JS, Kim SJ, Kornfeld H, et al. A chemoattractant cytokine associated with granulomas in tuberculosis and silicosis. Proc Natl Acad Sci 1997; 94: 6414-9.

18. Carlson I, Tognazzi K, Manseau EJ, Dvorak HF, Brown LF. Osteopontin is strongly expressed by histiocytes in granulomas of diverse etiology. Lab Invest 1997; 77: 103-8.

19. Sato K, Iwai A, Nakayama Y, Morimoto J, Takada A, Maruyama M, et al. Osteopontin is critical to determine symptom severity of influenza through the regulation of NK cell population. Biochem Biophys Res Commun 2012; 417: 274-9.

20. Morimoto J, Sato K, Nakayama Y, Kimura C, Kajino K, Matsui Y, et al. Osteopontin modulates the generation of memory CD8+ T cells during influenza virus infection. J Immunol 2011; 187: 5671-83.

21. Kadota J, Mizunoe S, Mito K, Mukae H, Yoshioka S, Kawakami K, et al. High plasma concentrations of osteopontin in patients with interstitial pneumonia. Respir Med 2005; 99: 111-7.

22. Vaschetto R, Nicola S, Olivieri C, Boggio E, Piccolella F, Mesturini R, et al. Serum levels of osteopontin are increased in SIRS and sepsis. Intensive Care Med 2008; 34: 2176-84.

23. van der Windt GJ, Hoogerwerf JJ, de Vos AF, Florquin S, van der Poll T. Osteopontin promotes host defense during Klebsiella pneumoniae-induced pneumonia. Eur Respir J 2010; 36: 1337-45.

24. Ueno T, Miyazaki E, Ando M, Nureki S, Kumamoto T. Osteopontin levels are elevated in patients with eosinophilic pneumonia. Respirology 2010; 15: 1111-21.

25. van der Windt GJ, Hoogendijk AJ, Schouten M, Hommes TJ, de Vos AF, Florquin S, et al. Osteopontin impairs host defense during pneumococcal pneumonia. J Infect Dis 2011; 203: 1850-8.

26. Mandell LA, Wunderink RG, Anzueto A, Bartlett JG, Campbell GD, Dean NC, et al. Infectious Diseases Society of America/American Thoracic Society consensus guidelines on the management of community-acquired pneumonia in adults. Clin Infect Dis 2007; 44 Suppl 2: S27-72.

27. Fine MJ, Auble TE, Yealy DM, Hanusa BH, Weissfeld LA, Singer DE, et al. A prediction rule to identify low-risk patients with community-acquired pneumonia. N Engl J Med 1997; 336: 243-50.

28. Knaus WA, Draper EA, Wagner DP, Zimmerman JE. APACHE II: a severity of disease classification system. Crit Care Med 1985; 13: 818-29.

29. Lim WS, van der Eerden MM, Laing R, Boersma WG, Karalus N, Town GI, et al. Defining community acquired pneumonia severity on presentation to hospital: an international derivation and validation study. Thorax 2003; 58: 377-82.

30. Shah BA, Ahmed W, Dhobi GN, Shah NN, Khursheed SQ, Haq I. Validity of pneumonia severity index and CURB-65 severity scoring systems in community acquired pneumonia in an Indian setting. Indian J Chest Dis Allied Sci 2010; 52: 9-17.

31. Brunkhorst FM, Al-Nawas B, Krummenauer F, Forycki ZF, Shah PM. Procalcitonin, C-reactive protein and APACHE II score for risk evaluation in patients with severe pneumonia. Clin Microbiol Infect 2002; 8: 93-100.

32. Thiem U, Niklaus D, Sehlhoff B, Stuckle C, Heppner HJ, Endres HG, et al. C-reactive protein, severity of pneumonia and mortality in elderly, hospitalised patients with community-acquired pneumonia. Age Ageing 2009; 38: 693-7.

33. Tateda $K$, Kusano $E$, Matsumoto $T$, Kimura $K$, Uchida $K$, Nakata $K$, et al. Semi-quantitative analysis of Streptococcus pneumoniae urinary antigen: kinetics of antigen titers and severity of diseases. Scandinavian journal of infectious diseases. 2006; 38: 166-71.

34. Viasus D, Del Rio-Pertuz G, Simonetti AF, Garcia-Vidal C, Acosta-Reyes J, Garavito A, et al. Biomarkers for predicting short-term mortality in community-acquired pneumonia: A systematic review and meta-analysis. J Infect 2016.

35. Chalmers JD, Singanayagam A, Hill AT. C-reactive protein is an independent predictor of severity in community-acquired pneumonia. Am J Med 2008; 121 : 219-25.

36. Hohenthal U, Hurme S, Helenius H, Heiro M, Meurman O, Nikoskelainen J, et al. Utility of C-reactive protein in assessing the disease severity and complications of community-acquired pneumonia. Clin Microbiol Infect 2009; 15: 1026-32.

37. Roderburg C, Benz F, Cardenas DV, Lutz M, Hippe HJ, Luedde T, et al. Persistently elevated osteopontin serum levels predict mortality in critically ill patients. Crit Care 2015; 19: 271.

38. Sheldon J, Riches P, Gooding R, Soni N, Hobbs JR. C-reactive protein and its cytokine mediators in intensive-care patients. Clin Chem 1993; 39: 147-50.

39. Lund SA, Giachelli CM, Scatena M. The role of osteopontin in inflammatory processes. J Cell Commun Signal 2009; 3: 311-22.

40. Harbarth S, Holeckova K, Froidevaux C, Pittet D, Ricou B, Grau GE, et al. Diagnostic value of procalcitonin, interleukin-6, and interleukin-8 in critically ill patients admitted with suspected sepsis. Am J Respir Crit Care Med 2001; 164: 396-402.

41. Kelly MM, Leigh R, Gilpin SE, Cheng E, Martin GE, Radford K, et al. Cell-specific gene expression in patients with usual interstitial pneumonia. Am J Respir Crit Care Med 2006; 174: 557-65.

42. Takahashi F, Takahashi K, Shimizu K, Cui R, Tada N, Takahashi H, et al. Osteopontin is strongly expressed by alveolar macrophages in the lungs of acute respiratory distress syndrome. Lung 2004; 182: 173-85.

43. Pardo A, Gibson K, Cisneros J, Richards TJ, Yang Y, Becerril C, et al. Up-regulation and profibrotic role of osteopontin in human idiopathic pulmonary fibrosis. PLoS Med 2005; 2: e251.

44. Simon L, Gauvin F, Amre DK, Saint-Louis P, Lacroix J. Serum procalcitonin and C-reactive protein levels as markers of bacterial infection: a systematic review and meta-analysis. Clin Infect Dis 2004; 39: 206-17.

45. Whicher J, Bienvenu J, Monneret G. Procalcitonin as an acute phase marker. Ann Clin Biochem 2001; 38: 483-93. 